\title{
Spatial-Temporal Modeling of Extreme Bottom-up Filling of Through-Silicon-Vias
}

\author{
D. Wheeler, ${ }^{\text {a T. P. Moffat, }}{ }^{\mathrm{b}, *}$ and D. Josell ${ }^{\mathrm{b}, \mathrm{z}}$ \\ ${ }^{a}$ Theiss Research, La Jolla, California 92037, USA \\ ${ }^{b}$ Materials Science and Engineering Division, Material Measurement Laboratory, National Institute of Standards \\ and Technology, Gaithersburg, Maryland 20899, USA
}

\begin{abstract}
Extreme bottom-up superfilling of annular through-silicon-vias (TSV) during copper electrodeposition has been reported wherein metal deposits on the bottom surface of the TSV with negligible deposition on its sidewalls or the field around it. The growth mode derives from a suppressor species that generates critical behavior manifesting as a negative differential resistance that, when coupled with a resistive electrolyte, yields bottom-up filling of recessed surface features. This paper models the observed phenomenon, the predictions capturing the full dynamics of the bottom-up filling process. The model considers the impact of the potential drop in the resistive electrolyte between the reference electrode and workpiece and the gradients of metal ion and rate-suppressing additive that accompany TSV filling. The predictions provide insight into the temporal and spatial variations of concentrations and overpotential that drive the bottom-up metal deposition process.
\end{abstract}

(C) 2013 The Electrochemical Society. [DOI: 10.1149/2.040312jes] All rights reserved.

Manuscript submitted October 1, 2013; revised manuscript received November 6, 2013. Published December 6, 2013. This paper is part of the JES Focus Issue on Electrochemical Processing for Interconnects.

Superconformal electrodeposition of $\mathrm{Cu}$ underlies the fabrication of modern interconnects that are ubiquitous in microelectronics. For sub-micrometer recessed surface features the Curvature Enhanced Accelerator Coverage (CEAC) mechanism has been shown to capture the salient morphological and kinetic aspects of the superconformal filling process from the incubation period and inception of accelerated deposition at the bottom corners to bottom up filling and overfill bump formation. ${ }^{1-7}$ Superconformal film growth is also used for filling of microvias and through-holes in circuit boards and represents a cutting edge process in the fabrication of through-silicon-vias (TSV) used in chip stacking. ${ }^{8-23}$ In addition to additive interactions relevant to on-chip metallization, understanding of deposition in such features requires that additional depletion effects associated with transport into deeper features be considered. ${ }^{20-23}$

Recently a potentiostatic process was detailed that yields highly localized $\mathrm{Cu}$ deposition and bottom-up filling of annular through silicon vias (TSV) based on a single suppressor additive. ${ }^{24}$ As shown in Fig. 1 deposition proceeds almost exclusively from the bottom of the TSV despite seed-layer metallization covering the entire via surface profile. The unusual growth dynamic could not be accounted for using existing CEAC or leveling shape change models. ${ }^{1-7,19-23,25-30}$ In the former case, there is no accelerating additive in the electrolyte but rather a combination of halide and polyether species that co-adsorb to form a layer that inhibits the metal deposition reaction. In the latter case, in contrast to traditional leveling type models, based on gradients of inhibitor additives ${ }^{25-30}$ that predict smoothly varying deposit thickness down the filling feature, the optimized experimental feature filling reveals almost exclusive bottom-up growth (Fig. 1). Experimental work indicated a correlation between extreme bottom-up filling and critical behavior that manifested as an additive-derived s-shaped negative differential resistance (S-NDR). ${ }^{24}$ Suppressor induced voltammetric hysteresis has been described previously, however its coupling with electrolyte resistivity as well as non-linear metal deposition kinetics was not considered. ${ }^{32}$ Thus an analytical model based on coupling additive derived S-NDR with the electrolyte resistance between the working and reference electrode was developed that was able to account for the highly non-linear localized deposition and extreme bottom-up feature filling observed experimentally. ${ }^{31}$ More generally, the differing time scales of the rapid electrical response that affects the double layer potential and the slow diffusion of the rate controlling inhibitor into the TSV are consistent with spatial-temporal mismatch associated with a number of electrochemical reaction-migration

*Electrochemical Society Fellow.

${ }^{\mathrm{z}}$ E-mail: daniel.josell@ nist.gov systems. ${ }^{33-35}$ The same S-NDR dynamics were proposed to underlie other superfilling processes such as of iron group metal deposition in damascene size features ${ }^{36-38}$ as well as bottom-up Au filling of TSV using a single additive, polyethylenimine, in a resistive sulfitebased electrolyte. ${ }^{39}$ The same idea can also quantitatively account for the "butterfly" filling profile reported for $\mathrm{Cu}$ deposition in throughholes. $^{8-10}$

TSV feature filling in critical S-NDR systems has also been examined under galvanostatic conditions where the local current density at the bottom of the features necessarily increases as the suppressor progressively blocks deposition on the field and upper reaches of the TSV. ${ }^{40}$ A formal model was able to predict superconformal filling, including both CEAC and extreme bottom-up filling modalities of localized deposition. Evolution of the growth front accompanying bottom-up growth dynamic was similar to that predicted by the fixed applied potential S-NDR model. ${ }^{31}$

The construct developed in this work adds full temporal evolution to the quantities and geometries previously approximated in the pseudo steady state model for fixed potential deposition. ${ }^{31}$ It also implements full radially symmetric 2D concentration fields for both the metal ion and additive in the electrolyte over and within the TSV. It maintains previously considered charge transfer kinetics for the metal deposition as a function of adsorbate coverage, accumulation and consumption/disruption of suppressing adsorbate during metal deposition and the important non-linear coupling between deposition current and potential distribution between the reference electrode and workpiece due to electrolyte resistivity.

\section{The Model}

The geometry considered is the annular TSV of the experimental results captured in Fig. 1. As in the simple 1-D model, the magnitude of the metal deposition velocity is linear in the coverage $\theta$ of adsorbate and the local metal ion concentration $\mathrm{C}_{\mathrm{Cu}}$ in the electrolyte. Normalizing to the bulk electrolyte concentration gives

$$
v_{\theta}=\frac{C_{C u}}{C_{C u}^{\infty}} v_{\theta=0}(1-\theta)
$$

where $\mathrm{v}_{\theta=0}$ corresponds to the suppressor-free metal deposition velocity. This linearization sets the deposition rate on the fully suppressed surface to zero consistent with the absence of significant deposition on the fully suppressed surface in the TSV experiments. The magnitude of the suppressor-free deposition velocity is expressed in terms of the 

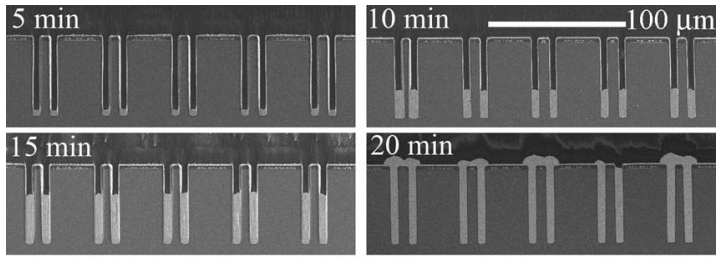

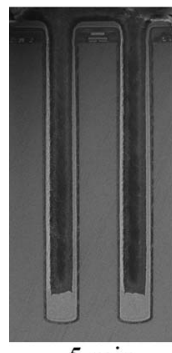

$5 \mathrm{~min}$

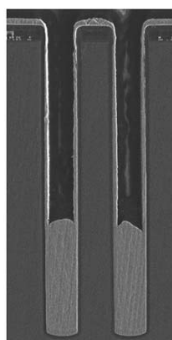

$10 \mathrm{~min}$

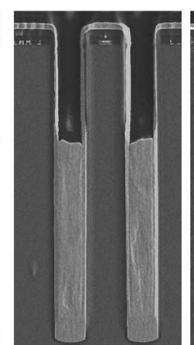

$15 \mathrm{~min}$

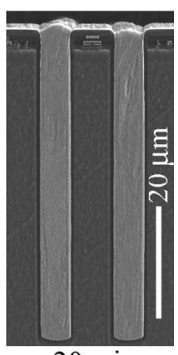

$20 \mathrm{~min}$
Figure 1. Cross-sectioned annular through silicon vias imaged by scanning electron microscope showing bottom-up filling during the electrodeposition of copper with negligible deposition on the metalized sidewalls or field over the features. (from Ref. 24)

forward and back reaction

$$
\nu_{\theta=0}=\nu^{o}\left(e^{\frac{-\alpha F \eta}{R T}}-e^{\frac{(2-\alpha) F \eta}{R T}}\right)
$$

with the growth velocity related to the deposition current density $\mathrm{j}$ by $\mathrm{j}=\mathrm{nFv} / \Omega$ for number of equivalents $\mathrm{n}$, Faraday's constant $\mathrm{F}$, and molar volume $\Omega ; \mathrm{v}$ is used interchangeably with $\mathrm{v}_{\theta}$ throughout this work. The overpotential $\eta$ that drives the deposition reaction at the metal/electrolyte interface is referenced to the reversible Nernst potential for the reaction. The potential applied to the workpiece, $\mathrm{E}_{\mathrm{App}}$, is distributed between the overpotential $\eta$ at the interface and the resistive (iR) losses associated with charge transport within the electrolyte; thus $\mathrm{E}_{\mathrm{App}}=\eta+\phi$, where $\phi$ equals the change of potential across the electrolyte from the reference electrode to the location on the workpiece. The reference electrode is assumed to have sufficient surface area that it exhibits negligible polarization.

The electrochemical cell is assumed to be a parallel plate workingcounter electrode arrangement separated by distance $L$ and electrolyte with resistivity $\rho$. For simplicity, the counter electrode also serves as the reference point in the present treatment. The model ensures electroneutrality within the electrolyte is maintained by imposing

$$
\nabla^{2} \phi=0
$$

while the current density $j$ in the electrolyte is given by ohms law

$$
\vec{j}=-\frac{1}{\rho} \nabla \phi
$$

In the schematic geometry shown in Fig. $2, \nabla \phi$ is required to be parallel to the cell wall on the sides of the cell (zero normal flux condition). The local current density at the deposit surface, related to $\eta$ by Eq. 2 (and thus to $\phi$ ), is equated to the $\mathrm{Cu}^{2+}$ flux onto the deposit (surface normal $\hat{n}$ ) according to

$$
\frac{1}{2 F} \vec{j} \cdot \hat{n}=-D_{C u} \nabla C_{C u} \cdot \hat{n}
$$

A capacitive charging term for the double layer, while included in the numerical model, is omitted for clarity because it has no significant impact beyond the first few milliseconds. The equations defining the metal ion distribution $\mathrm{C}_{\mathrm{Cu}}$ follow shortly. To accelerate the computations, the potential distribution was solved within the boundary layer and feature but a uniform gradient was assumed from the reference to the boundary layer. The overpotential $\eta$ available for driving the deposition reaction at a given point on the working electrode is obtained using $\mathrm{E}_{\mathrm{App}}-\phi$ at that location. As anticipated in the 1-D model the

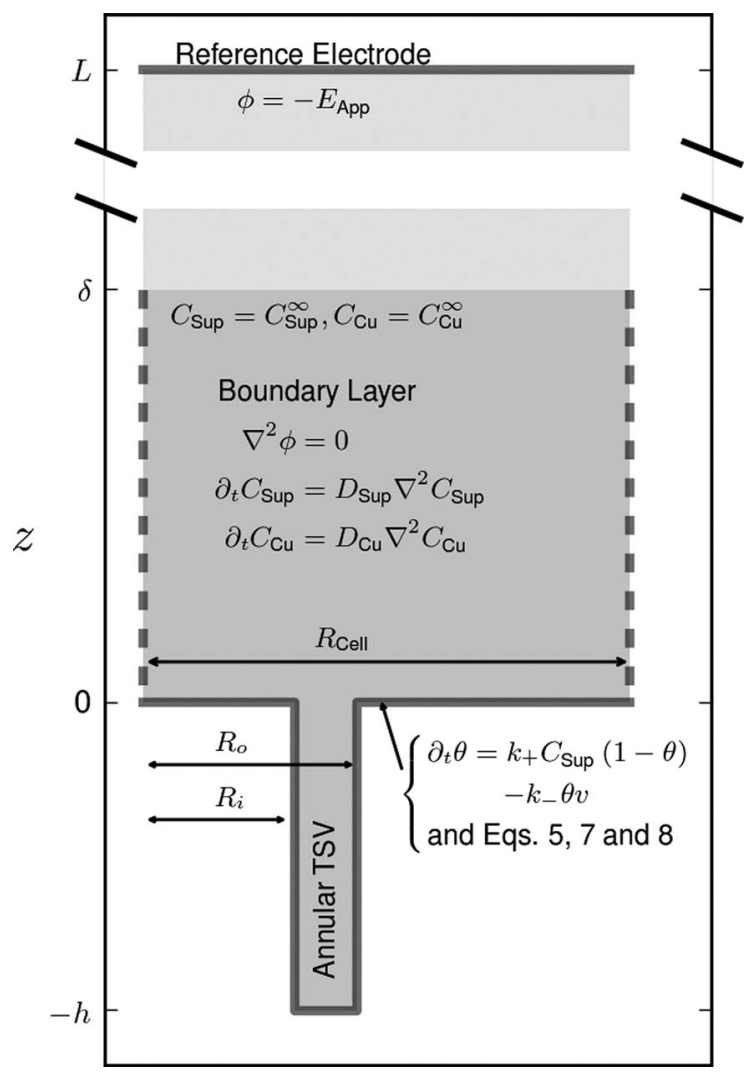

Figure 2. Schematic of geometry used to model feature filling along a radial cut in the annular TSVs. Transport in the boundary layer and unfilled volume of the TSV are modeled assuming Fickian diffusion within the time- and positiondependent concentration fields. The metal deposition rate and suppressor adsorption kinetics are as defined, the adsorbate coverage evolving through both adsorption and consumption, with the boundary conditions applying to the moving surfaces. Parameters are defined in Table I.

ohmic losses within the TSV are much smaller than those across the much larger distance between the reference electrode and workpiece.

Evolution of the metal ion $\mathrm{C}_{\mathrm{Cu}}$ and suppressor $\mathrm{C}_{\text {Sup }}$ concentrations and associated adsorbate coverage $\theta(\mathrm{z})$ distributions within the filling feature are defined by the standard bulk diffusion equations

$$
\frac{\partial C_{C u}}{\partial t}=D_{C u} \nabla^{2} C_{C u} \quad \text { and } \quad \frac{\partial C_{S u p}}{\partial t}=D_{S u p} \nabla^{2} C_{S u p}
$$

Bulk concentrations are imposed at the top of the boundary layer and concentration gradients and zero flux conditions are imposed on the sides of the cell. Boundary conditions on the deposit are

$$
-D_{C u} \nabla C_{C u} \cdot \hat{n}=\frac{\nu}{\Omega}
$$

and

$$
-D_{\text {Sup }} \nabla C_{\text {Sup }} \cdot \hat{n}=C_{\text {Sup }} k_{+} \Gamma(1-\theta)
$$

Equation 7 equates the metal ion flux to the local deposition rate $v_{\theta}$ using the molar volume $\Omega$ of the metal. Equation 8 equates the flux of suppressor from the electrolyte to its rate of adsorption on the deposit surface with an adsorption rate constant $\mathrm{k}_{+}$and saturation coverage $\Gamma$. It assumes that the adsorption occurs at a rate proportional to the local suppressor concentration in the adjacent electrolyte with the fraction of free sites being $1-\theta$. A more sophisticated model might explicitly consider the contribution of migration to the $\mathrm{Cu}^{2+}$ transport across the hydrodynamic boundary layer in terms of the Nernst-Planck equation.

Evolution of the fractional coverage of the suppressing adsorbate $\theta$ is determined by the balance between its accumulation from the electrolyte and its consumption. The deactivation or consumption of the inhibiting species is assumed to be proportional to the metal 
Table I. Values for feature geometry, ion and additive transport, and deposition and attachment kinetics are listed for all parameters used in this study. Values used for the simulations are as indicated in this table unless otherwise specified.

\begin{tabular}{llll} 
Parameter & Name & Units & \multicolumn{1}{c}{ Value } \\
\hline Simulation cell radius & $\mathrm{R}_{\text {Cell }}$ & $\mathrm{m}$ & $28 \times 10^{-6}$ \\
Outer radius of annular TSV & $\mathrm{R}_{\mathrm{o}}$ & $\mathrm{m}$ & $8.5 \times 10^{-6}$ \\
Inner radius of annular TSV & $\mathrm{R}_{\mathrm{i}}$ & $\mathrm{m}$ & $4 \times 10^{-6}$ \\
TSV height & $\mathrm{h}$ & $\mathrm{m}$ & $56 \times 10^{-6}$ \\
Distance to reference electrode & $\mathrm{L}$ & $\mathrm{m}$ & 0.03 \\
Diffusion coefficient, copper & $\mathrm{D}_{\mathrm{Cu}}$ & $\mathrm{m}^{2} / \mathrm{s}$ & $2.65 \times 10^{-10}$ \\
Diffusion coefficient, suppressor & $\mathrm{D}_{\mathrm{Sup}}$ & $\mathrm{m}^{2} / \mathrm{s}$ & $9.2 \times 10^{-11}$ \\
Bulk concentration, copper & $C_{\mathrm{Cu}}^{\infty}$ & $\mathrm{mol} / \mathrm{m}^{3}$ & 1000 \\
Electrolyte resistivity & $\rho$ & $\Omega \mathrm{m}$ & 0.0655 \\
Boundary layer thickness & $\delta$ & $\mathrm{m}$ & $150 \times 10^{-6}$ \\
Cu deposition rate constant & $\mathrm{v}^{\mathrm{o}}$ & $\mathrm{m} / \mathrm{s}$ & $7.73 \times 10^{-8}$ \\
Saturation suppressor coverage & $\Gamma$ & $\mathrm{mol} / \mathrm{m}^{2}$ & $2.5 \times 10^{-7}$ \\
Deposition kinetic factor & $\alpha$ & - & 0.4 \\
Copper ionic charge & $\mathrm{n}$ & - & 2 \\
Copper molar volume & $\Omega$ & $\mathrm{m}^{3} / \mathrm{mol}$ & $7.1 \times 10^{-6}$
\end{tabular}

deposition rate and the fractional coverage of the adsorbed suppressor. This coverage evolution is modeled as

$$
\frac{d \theta}{d t}=k_{+} C_{S u p}(1-\theta)-k_{-} \theta v_{\theta}
$$

The full system of equations was solved numerically in a cylindrical cell of radius $\mathrm{R}_{\mathrm{Cell}}$ extending from the workpiece to the plane of the reference. A level set approach was used to evaluate the concentration and electrostatic potential fields within the unfilled regions in and over the TSV and adsorbate coverage on the deposit surface consistent with the stationary and moving boundary conditions (as in Refs. 3, 41, and 42). The simulations presented are the result of systematic variation of mesh size, time increment and nonlinear solver tolerance to ensure convergence of the data presented herein.

\section{Model Results}

Unless stated otherwise, values for the parameters used in the simulations are given in Table I. Concentrations and dimensions correspond to experimental conditions relevant to the annular TSV in the experimental study of $\mathrm{Cu}$ extremefill. ${ }^{24}$ The values are representative of the experimental annular vias shown in Fig. 1: $56 \mu \mathrm{m}$ deep with outer radius $\mathrm{R}_{\mathrm{o}} \approx 9.5 \mu \mathrm{m}$ and inner radius $\mathrm{R}_{\mathrm{i}} \approx 4 \mu \mathrm{m}$. Modeling assumes cylindrical symmetry with a no-flux border placed at a radius of $50 / \sqrt{\pi} \mu \mathrm{m}$, the same area per feature as in the experimental study (50 $\mu \mathrm{m}$ via spacing) and the simple model. Figure 2 shows a schematic of the TSV geometry with concentration and potential fields and associated boundary conditions indicated. Parameters for the transport and kinetics are drawn from the literature where possible. ${ }^{24}$

Figure 3 shows filling of the annular TSV predicted by the model over an array of $\mathrm{k}_{+}$and $\mathrm{k}_{-}$values for two combinations of $\mathrm{C}_{\text {Sup }}$ and $\mathrm{E}_{\mathrm{App}}$. In both cases deposition is nonlocalized for large $\mathrm{k}_{-}$and small $\mathrm{k}_{+}$; keyhole voiding occurs due to the associated metal ion depletion. Complete suppression of deposition is predicted for small $\mathrm{k}_{-}$ and large $\mathrm{k}_{+}$, aside from limited deposition at the TSV bottom in the first several minutes of the initial transient. Deposition is localized to the bottom of the feature with bottom-up TSV filling for intermediate $\mathrm{k}_{-}$and $\mathrm{k}_{+}$, qualitatively capturing the evolution of feature filling observed experimentally (Fig. 1). Where complete suppression is predicted, it occurs within approximately $200 \mathrm{~s}$. Where deposition does not localize to yield bottom-up filling of the feature, pinch-off with resulting void formation occurs within approximately $200 \mathrm{~s}$ to $600 \mathrm{~s}$ (larger voids form given a shorter time to pinch-off). Defect-free filling takes approximately $2000 \mathrm{~s}$. Coupling suppressor adsorption and disruption with current constraint through finite electrolyte resistance thus provides a mechanism for extreme bottom-up filling of recessed

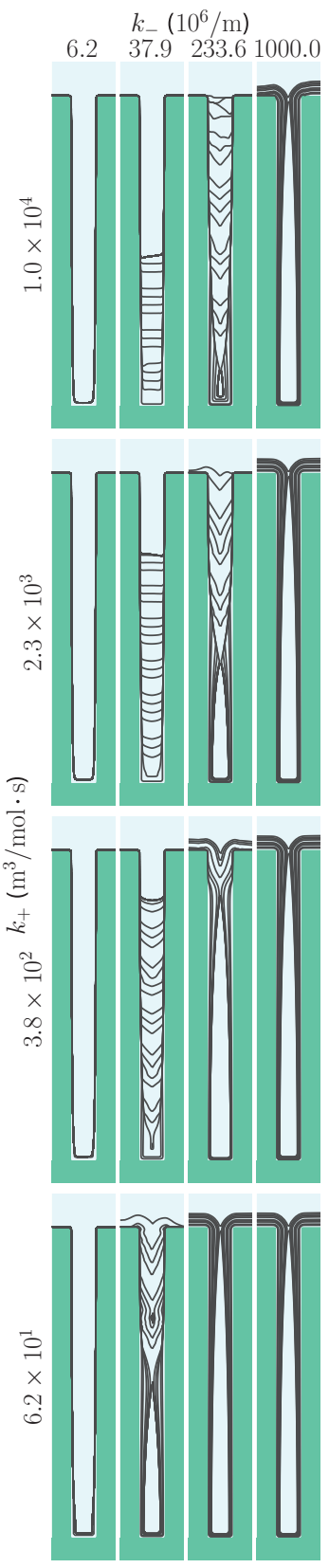

(a)
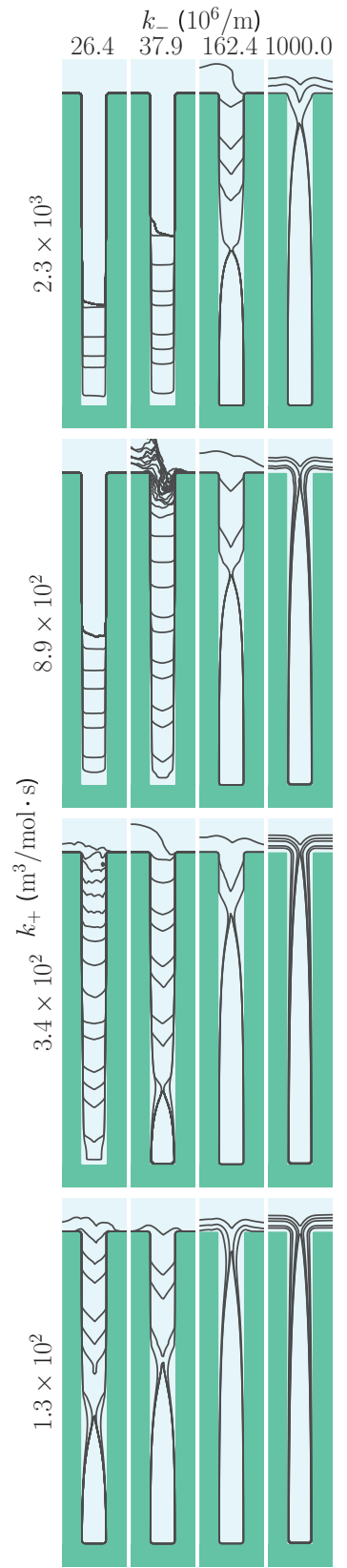

(b)
Figure 3. (a) Filling contours along a radial cut from the annular TSV predicted by the model for specified values of the adsorption kinetics $\mathrm{k}_{+}$and consumption kinetics $\mathrm{k}_{-}$using $\mathrm{C}_{\text {Sup }}=0.02 \mathrm{~mol} / \mathrm{m}^{3}$ and $\mathrm{E}_{\mathrm{App}}=-0.25 \mathrm{~V}$. b) Analogous simulations for $\mathrm{C}_{\text {Sup }}=0.06 \mathrm{~mol} / \mathrm{m}^{3}$ and $\mathrm{E}_{\mathrm{App}}=-0.45 \mathrm{~V}$. Other parameters are indicated in Table I. In both cases, after a short period of deposition localized to the TSV bottom, complete suppression of feature filling is predicted for small $\mathrm{k}_{-}$and large $\mathrm{k}_{+}$. Deposition is nonlocalized for large $\mathrm{k}_{-}$ and small $\mathrm{k}_{+}$; keyhole voiding occurs due to the associated metal ion depletion. Deposition is localized to the bottom of the feature with bottom-up TSV filling for intermediate $\mathrm{k}_{-}$and $\mathrm{k}_{+}$.

surface features. Figure 4 explicitly shows the annular geometry for a prediction with defect-free filling falling within the range of parameters examined in Fig. 3.

Several of the simulations in Fig. 3 exhibit bottom-up deposition that halts before complete filling of the TSV, suppression reasserting itself due to insufficient decrease of suppressor concentration down the still unfilled region. Albeit this behavior was not observed experimentally, it is consistent with pseudo steady state predictions that localized 


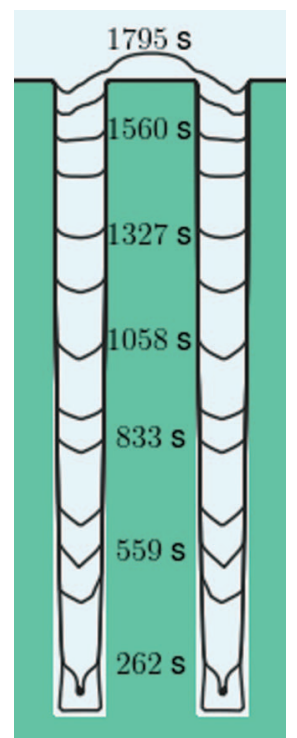

Figure 4. Simulation of TSV filling for $\mathrm{C}_{\text {Sup }}=0.06 \mathrm{~mol} / \mathrm{m}^{3}, \mathrm{E}_{\mathrm{App}}=-0.45 \mathrm{~V}$, $\mathrm{k}_{+}=546 \mathrm{~m}^{3} / \mathrm{mol} \cdot \mathrm{s}$ and $\mathrm{k}_{-}=3.79 \times 10^{7} 1 / \mathrm{m}$ explicitly showing the annular geometry as viewed in cross-section.

bottom-up deposition might not occur in sufficiently shallow features. The conditions for sustained filling observed experimentally might also reflect some aspect of adsorbate disruption that is not explicitly accounted for in Eq. 9. ${ }^{24}$ Likewise the contribution of migration to enhanced $\mathrm{Cu}$ flux as the growth front nears the top of the TSV, transitioning from sampling the rectilinear to hemispherical fields, might need to be considered. ${ }^{43,44}$

Predicted evolution of the deposition rate and suppressor coverage (at the TSV bottom, midway between $\mathrm{R}_{\mathrm{i}}$ and $\mathrm{R}_{\mathrm{o}}$ ) and the overpotential driving deposition (evaluated at the top corner of the TSV) are shown in Fig. 5 for representative examples from Fig. 3a (the first three simulations in the third row). Time-dependent variation of the overpotential driving deposition on the workpiece, due to variation of the potential drop from the reference to the workpiece, is central to the extreme feature filling dynamic in the constant applied potential process. Where the entire surface is in the active state, true at the earliest times shown for all cases, the ohmic losses associated with the current flow reduce the overpotential $\eta$ to only $\approx-0.03 \mathrm{~V}$, far less than the applied value of $-0.25 \mathrm{~V}$. The overpotential remains low for the entire subconformal deposition process due to the current drawn by the large active surface area. In contrast, where localized deposition arises after the initial transient, increase of overpotential over time through the progressive suppression of deposition on the field and down the TSV is seen; as this area passivates, the increasing overpotential stabilizes deposition on as-yet unpassivated surfaces. The nature of the deposition also impacts the deposition rate on the bottom surface; localized deposition yields fast, bottom-up growth while non-localized deposition yields slower growth at the bottom. Suppressor coverage drops rapidly (everywhere) for nonlocalized deposition but more slowly (and only on the TSV bottom) for localized deposition.

Figure 6 summarizes the TSV filling behavior predicted by the model for a range of values of the adsorption and consumption kinetics $\mathrm{k}_{+}$and $\mathrm{k}_{-}$. The first map indicates the maximum height to which the metal fills the TSV (as a fraction of the TSV height). Shutdown of deposition associated with early passivation is evident when the suppressor accumulates more rapidly than it can be consumed (blue region). The second map shows the height of occluded voids within this metal deposit (also scaled by the TSV height). Their presence is generally indicative of nonlocalized deposition and associated pinchoff (red region). The third map gives the difference between these values; a value of unity (red region) indicates a defect-free, fully filled TSV. The trend of behavior, including the diagonal band of superior filling with increasing $\mathrm{k}_{+}$and $\mathrm{k}_{-}$, is quite similar to the band of conditions predicted by the pseudo steady state model to yield extremefill (despite that model's inability to capture history dependence implicit in the hysteretic nature of the S-NDR). ${ }^{31}$

The suppressor concentration $\mathrm{C}_{\text {Sup }}$ and applied potential $\mathrm{E}_{\mathrm{App}}$ are readily controlled parameters that strongly influence feature filling. Maps analogous to those in Fig. 6 are shown in Fig. 7 for variation of these parameters. Two experimental conditions that were shown to generate bottom-up filling ${ }^{24,31}$ as exemplified in Fig. 1 are indicated. The model predicts localized bottom-up filling for applied potential and suppressor concentrations where such behavior was observed experimentally. Total feature filling is, however, only predicted to occur for conditions differing somewhat from those used experimentally; the absence of data regarding a number of the modeling parameters, including in the kinetics for suppressor adsorption and consumption, likely underlies the difference.

\section{Discussion}

The temporal evolution of experimentally observed extreme bottom-up $\mathrm{Cu}$ filling of TSVs is captured by the model convolving
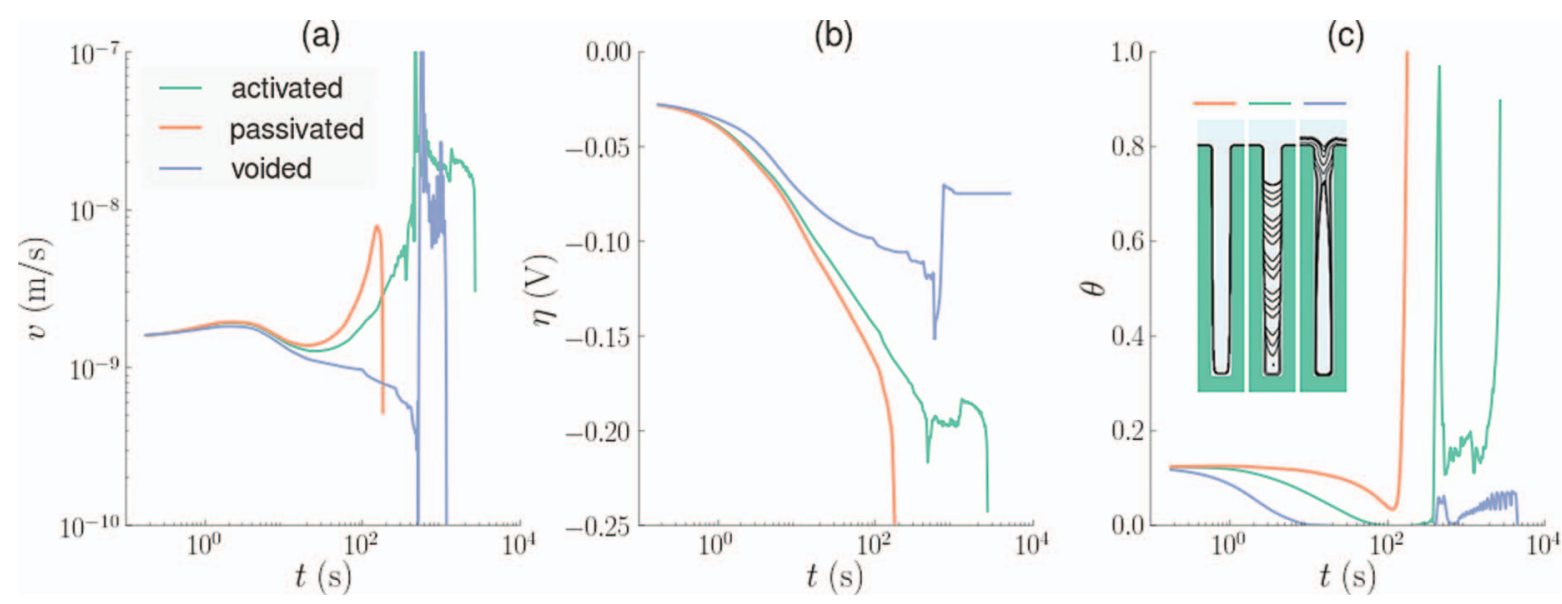

Figure 5. Predicted time-dependence of deposition rate, overpotential and suppressor coverage for examples of passivation, bottom-up deposition (incomplete filling) and nonlocalized deposition with voiding corresponding to the first three simulations in the third row of Fig. 3a. a) Evolution of the deposition rate from the TSV bottom midway between $R_{i}$ and $R_{o}$. b) Evolution of the overpotential $\eta$ evaluated at the upper edge of the TSVs. c) Evolution of the suppressor coverage midway between $R_{i}$ and $R_{0}$. The results for the voided fill reflect deposition above the void after $\approx 500 \mathrm{~s}$. The filling contours, copied from Fig. 3a, are overlaid in c). 
(a)

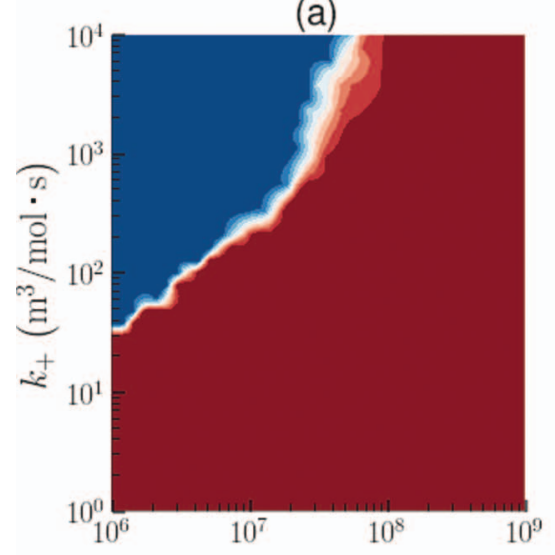

(b)

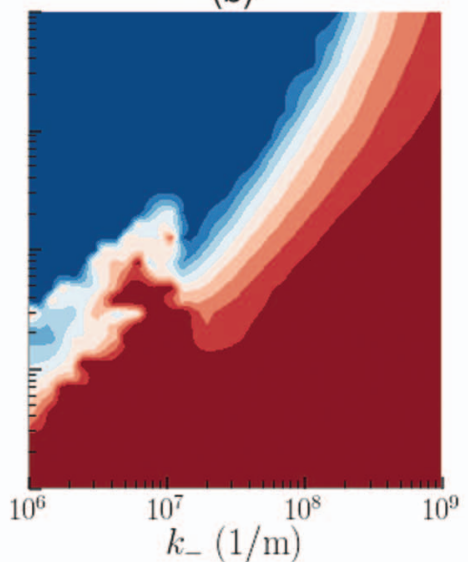

(c)

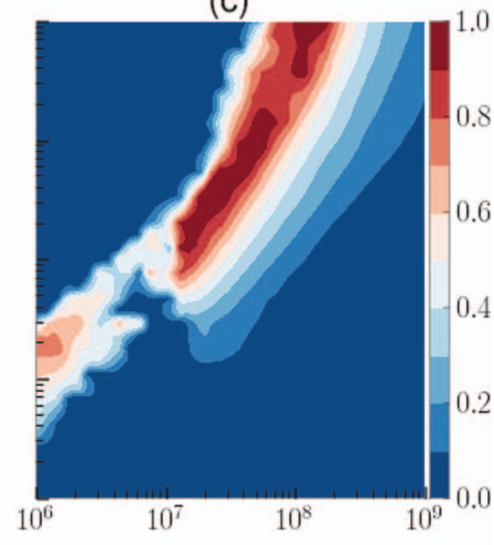

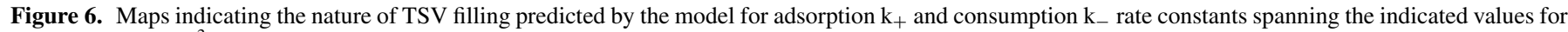

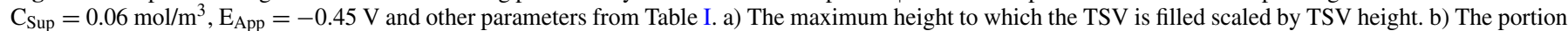

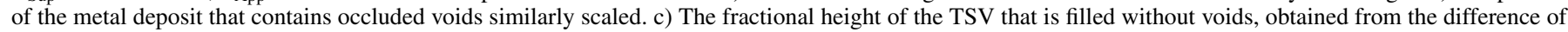
the values in the preceding maps. Defect-free TSV filling is associated with unity value.

positive feedback associated with metal deposition induced breakdown of a suppressor layer with evolution of the overpotential at the work piece. The different time scales for electrical response and mass transport are seen to enable the system to effectively bifurcate into active and passive region, deposition in the active regions being localized to the bottoms of the TSVs. ${ }^{24}$ When the field is initially fully active the current associated with uninhibited deposition results in ohmic losses in the electrolyte that greatly constrain the reaction overpotential at the workpiece. This enables rapid passivation of the free surface through accumulation of the arriving suppressor. The resulting attenuation of the total current flowing in the system reduces the associated ohmic losses, allowing the overpotential to increase toward the applied value. This stimulates on-going metal deposition on surfaces farther down the TSV that remain un-passivated due to the diffusion limited suppressor transport and adsorption as well as disruption of adsorbate coverage by the rapid metal deposition itself. The topology of the substrate dictates the locations of the active regions and thus effectively becomes a design parameter that makes an otherwise unstable deposition process manufacturable. The full time-dependent solution thus confirms the mechanism of extremefill originally proposed with the pseudo steady state model. ${ }^{31}$

Extreme bottom-up filling is anticipated for a range of conditions, the predicted behavior reasonably consistent with results reported for $\mathrm{Cu}$ TSV filling. The mechanism and model should be widely applicable to electrochemical deposition across a range of chemistries and applications.

\section{Conclusions}

A solution of time-dependent TSV filling has been presented that predicts deposition localized to the bottoms of filling features under potentiostatic control; the construct captures the salient aspects of experimentally observed extreme bottom-up filling during copper electrodeposition in TSVs. The filling mechanism involves transport constrained adsorption of a suppressing additive within the recessed surface and its disruption by the metal deposition reaction that together give rise to positive feedback. Concurrent dynamic redistribution of applied potential between the reaction overpotential and the resistive (a)

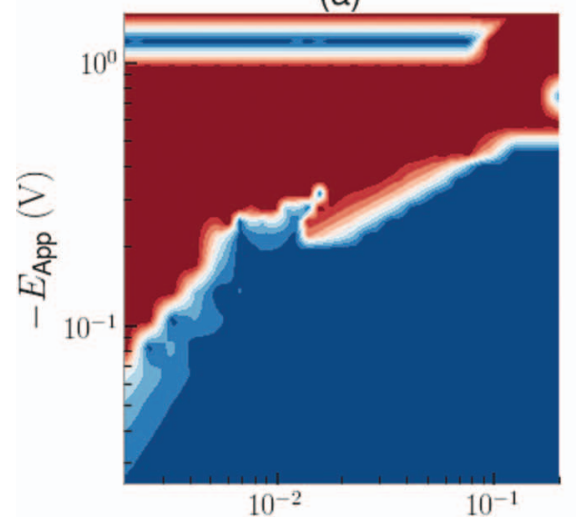

(b)

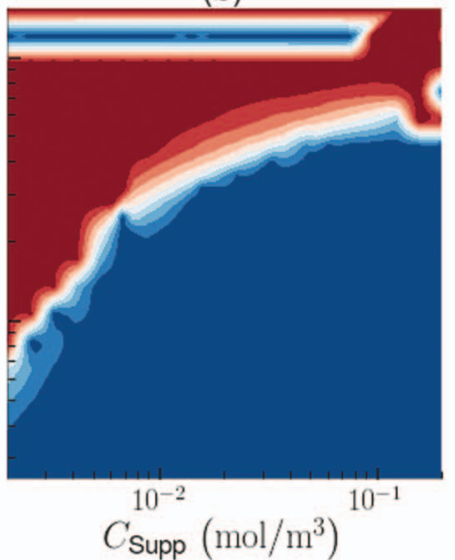

(c)

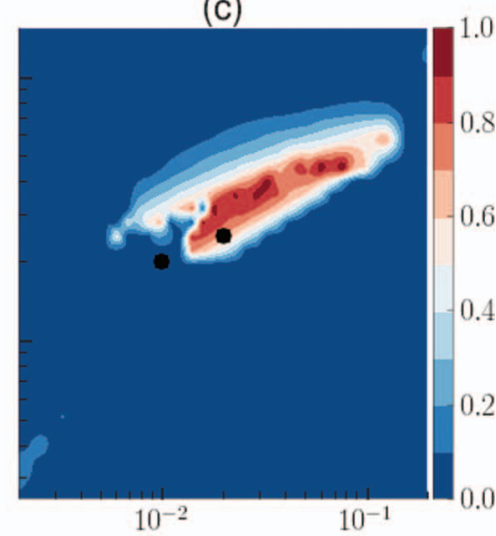

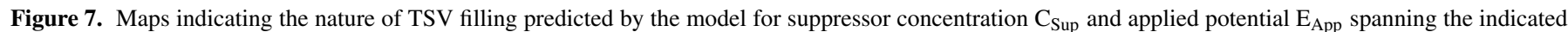

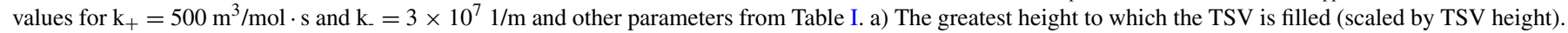

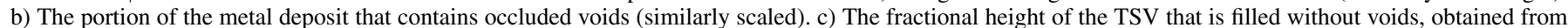

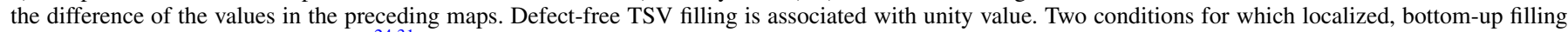
with $\mathrm{Cu}$ was experimentally observed ${ }^{24,31}$ are indicated. 
electrolyte results in passivation of the free surface and upper sidewalls with the metal deposition localized to the bottom of the features. The results are consistent with experimental observations of $\mathrm{Cu}$ electrodeposition in annular TSV as well as predictions of a simpler model based on a pseudo steady state solution of the problem. The mechanism requires little more than a resistive electrolyte and a ratesuppressing additive; as suggested by experimental results with other systems, the possibility for broad application to other materials and systems is therefore considerable.

\section{Acknowledgments}

D. Wheeler and Theiss Research acknowledge support from NIST grant 70NANB12H196. The authors also thank Dan Edelstein of IBM T.J. Watson Research Laboratory for kindly providing the TSV patterned wafer used in the feature filling study.

\section{References}

1. T. P. Moffat, D. Wheeler, W. H. Huber, and D. Josell, Electrochem. Solid-State Lett. 4, C26 (2001).

2. D. Josell, D. Wheeler, W. H. Huber, J. E. Bonevich, and T. P. Moffat, J. Electrochem. Soc., 148, C767 (2001).

3. D. Wheeler, D. Josell, and T. P. Moffat, J. Electrochem. Soc., 150, C302 (2003).

4. T. P. Moffat, D. Wheeler, M. Edelstein, and D. Josell, IBM J. Res. and Devel., 49, 19 (2005).

5. D. Josell, D. Wheeler, and T. P. Moffat, J. Electrochem. Soc., 153, C11 (2006).

6. T. P. Moffat, D. Wheeler, S.-K. Kim, and D. Josell, Electrochimica Acta, 53, 145 (2007).

7. D. Josell, T. P. Moffat, and D. Wheeler, J. Electrochem. Soc., 154, D208 (2007).

8. W.-P Dow, D.-H. Liu, C.-W. Lu, C.-H. Chen, J. J. Yan, and S.-M. Huang, Electrochem and Solid-State Lett., 14, D13 (2011).

9. C. H. Chen, C.-W. Lu, S.-M. Huang, and W. P. Dow, Electrochimica Acta, 56, 5954 (2011).

10. W.-P. Dow, C.-W. Lu, J.-Y. Lin, and F. C. Hsu, Electrochem. Solid-State Lett., 14, D63 (2011).

11. O. Luhn, A. Radisic, P. M. Vereecken, C. Van Hoof, W. Ruythooren, and J.-P. Celis, Electrochem. Solid-State Lett., 12, D39 (2009).

12. O. Luhn, A. Radisic, C. Van Hoof, W. Ruythooren, and J.-P. Celis, J. Electrochem. Soc., 157, D242 (2010).
13. A. Radisic, O. Luh, H. G. G. Philipsen, Z. El-Mekki, M. Honore, S. Rodet, S. Armini, C. Drijbooms, H. Bender, and W. Ruythooren, Micrelectronic Engineering, 88, 701 (2011).

14. M. Hayase and K. Otsubo, J. Electrochem. Soc., 157, D628 (2010)

15. T. Hayashi, K. Kondo, T. Saito, M. Takeuchi, and N. Okamoto, J. Electrochem. Soc., 158, D715 (2011).

16. T. H. Tsai and J. H. Huang, Microelectronic Engineering, 88, 195 (2011).

17. C. Fang, A. L. Corre, and D. Yon, Microelectronic Engineering, 88, 749 (2011)

18. S. K. Cho, M. J. Kim, and J. J. Kim, Electrochem. Solid-State Lett., 14, D52 (2011).

19. T. Matsuoka, K. Otsubo, Y. Onishi, K. Amaya, and M. Hayase, Electrochimica Acta, 82, 356 (2012).

20. A. Pohjoranta and R. Tenno, J. Electrochem. Soc., 154, D502 (2007)

21. R. Tenno and A. Pohjoranta, J. Electrochem. Soc., 155, D383 (2008).

22. R. Akolkar and U. Landau, J. Electrochem. Soc., 151, C702 (2004).

23. R. Akolkar and U. Landau, J. Electrochem. Soc., 156, D351 (2009).

24. T. P Moffat and D. Josell, J. Electrochem. Soc., 159, D208 (2012).

25. Y. Cao, P. Taephaisitphongse, R. Chalupa, and A. C. West, J. Electrochem. Soc. 148 , C466 (2001).

26. J. O. Dukovic and C. W. Tobias, J. Electrochem. Soc., 137, 3748 (1990).

27. C Madore and D. Landolt, J. Electrochem. Soc., 143, 3936 (1996).

28. C. Madore, M. Matlosz, and D. Landolt, J. Electrochem. Soc., 143, 3927 (1996).

29. A. C. West, J. Electrochem. Soc., 147, 227 (2000).

30. R. Chalupa, Y. Cao, and A. C. West, J. Appl. Electrochem., 32, 135 (2002).

31. D. Josell, D. Wheeler, and T. P. Moffat, J. Electrochem. Soc., 159, D570 (2012).

32. D. Roha and U. Landau, J. Electrochem. Soc., 137, 824 (1990).

33. N. Mazouz and K. Krischer, J. Phys. Chem. B, 104, 7545 (2000).

34. Y.-J. Li, J. Oslonovitch, N. Mazouz, F. Plenge, K. Krischer, and G. Ertl, Science, 291, 2395 (2001).

35. K. Krischer, J. Electroanal. Chem., 501, 1 (2001).

36. S.-K. Kim, J. E. Bonevich, D. Josell, and T. P. Moffat, J. Electrochem. Soc., 154, D443 (2007).

37. C. H. Lee, J. E. Bonevich, J. E. Davies, and T. P. Moffat, J. Electrochem. Soc., 155, D499 (2008).

38. C. H. Lee, J. E. Bonevich, J. E. Davies, and T. P. Moffat, J. Electrochem. Soc., 156, D301 (2009).

39. D. Josell and T. P. Moffat, Journal of the Electrochemical Society, 160(12), D3035 (2013).

40. L. Yang, A. Radisic, J. Deconinck, and P. M. Vereecken, J. Electrochem. Soc., 160, D3051 (2013).

41. J. E. Guyer, D. Wheeler, and J. A. Warren, Computing in Science \& Engineering, 11, 6 (2009).

42. Code and additional details regarding the simulations can be found at https://github.com/wd15/extremefill2D.

43. C. Amatore, M. R. Deakin, and R. M. Wightman, J. Electroanal. Chem., 225, 49 (1987).

44. C. Amatore, A. Oleinick, and I. Svir, Anal. Chem., 80, 7947 (2008). 